INTESTINAL IMMUNOLOGY

\title{
Impairment of intestinal intraepithelial lymphocytes in Id2 deficient mice
}

\author{
J-K Kim, M Takeuchi, Y Yokota
}

Gut 2004;53:480-486. doi: 10.1136/gut.2003.022293

See end of article for authors' affiliations .....................

Correspondence to: Professor Y Yokota, Department of Molecular Genetics, School of Medicine, University of Fukui, 23-3 Shimoaizuki, Matsuoka, Fukui 910-1193, Japan; yyokota@fmsrsa. fukui-med.ac.jp

Accepted for publication 1 November 2003 lix transcription factors, regulates cell differentiation. Background: Id2, an inhibitor of basic helix-loop-helix transcription
$\mathrm{ld} 2^{-}-$mice exhibit a variety of phenotypes in the immune system.

Aims: In this study we investigated whether Id2 plays a role in intestinal intraepithelial lymphocytes (IELs), which constitute the main defence against pathogens in the intestinal tract.

Methods: Flow cytometry and bone marrow transplantation were used to analyse and characterise subsets of IELs of Id $2^{-/-}$mice. Gene expression was analysed by real-time polymerase chain reaction. Intestinal barrier function was evaluated by treating mice with 5 -fluorouracil (5-FU).

Results: Among the four members of the Id gene family, Id 2 was selectively expressed in all T cell subsets in the small intestinal IELs. Id $2^{-/-}$mice showed alteration in the proportions of $\mathrm{T}$ cell subsets and a substantial reduction in the number of IELs, especially those of the CD4 $4^{+}$and $C D 8 \alpha \beta^{+} T$ cell subsets, indicating a more pronounced effect on thymus derived IELs. Expression of $\alpha_{E}$ integrin was reduced in $\mathrm{CD} 4^{+}$and $\mathrm{CD} 8 \alpha \beta^{+}$T cell subsets in IELs of Id $2^{-/-}$mice. IELs isolated from C57BL/6 mice reconstituted with $\mathrm{Id} 2^{-/-}$bone marrow cells showed a similar phenotype to that of $\mathrm{Id} 2^{-/-}$mice, indicating that the defects are intrinsic to bone marrow derived cells. Expression of genes encoding intestinal epithelial cell derived cytokines was reduced in $\mathrm{ld} 2^{-/-}$mice. The 5 - FU treatment revealed impaired intestinal barrier function of ld $2^{-1-}$ mice.

Conclusions: The Id 2 gene is essential for constituting the intestinal mucosal barrier, particularly with respect to IELs. Id2 null mutant mice may provide a good experimental model for studying the ontogeny of IELs and intestinal inflammation and infection.
$\mathrm{T}$ he intestinal mucosal barrier, which consists of intestinal epithelial cells (IECs) and intestinal intraepithelial lymphocytes (IELs), is the first line of the host immune defence in the gut. IELs constitute a major lymphocyte population residing in close proximity to the intestinal lumen, ${ }^{1-3}$ and the size of the IEL population is equivalent to, or larger than, the population of peripheral lymphocytes in the spleen. ${ }^{1}$ These cells have been considered to comprise several ontogenetically and phenotypically distinct $\mathrm{T}$ cell subpopulations that can be identified by their expression of $\mathrm{T}$ cell receptor (TCR), CD4 and CD8: TCR $\alpha \beta^{+} \mathrm{CD} 4^{+} \mathrm{T}$ cells, CD $8 \alpha \beta^{+} \mathrm{T}$ cells expressing TCR $\alpha \beta$, and CD $8 \alpha \alpha^{+} \mathrm{T}$ cells expressing TCR $\alpha \beta$ or TCR $\gamma \delta$, together with minor subpopulations of TCR $\alpha \beta^{+} \mathrm{CD} 4^{+} \mathrm{CD} 8 \alpha \alpha^{+}$and TCR $\gamma \delta^{+} \mathrm{CD} 4^{-} \mathrm{CD} 8^{-} \mathrm{T}^{-}$cells. ${ }^{2}$ Among these T cells, CD $8 \alpha \alpha^{+} \mathrm{T}$ cells are unique to the IELs as they are not detected in conventional peripheral lymphoid organs such as the spleen, lymph nodes, and Peyer's patches. ${ }^{2-4}$ In addition, CD $8 \alpha \alpha^{+}$IELs do not recirculate in the periphery whereas TCR $\alpha \beta^{+} \mathrm{CD} 4^{+}$and $\mathrm{TCR} \alpha \beta^{+} \mathrm{CD} 8 \alpha \beta^{+} \mathrm{T}$ cells may represent circulating CD4 and CD8 $\alpha \beta$ T cells. ${ }^{5}$ Thus IELs are developmentally categorised into two groups: thymically derived TCR $\alpha \beta^{+} C D 4^{+}$and TCR $\alpha \beta^{+} \mathrm{CD} 8 \alpha \beta^{+} \mathrm{T}$ cells, and extrathymically derived $\operatorname{CD} 8 \alpha \alpha^{+} \mathrm{T}$ cells, which may differ with respect to their use of different cytokine signalling pathways to regulate their differentiation..$^{3-5}$

IELs have a number of important immunological functions, such as cytotoxic activity, secretion of cytokines, including interleukin (IL)-2, IL-3, IL-5, tumour necrosis factor $\alpha$, transforming growth factor $\beta$ (TGF- $\beta$ ), and interferon $\gamma($ IFN- $\gamma)$, and modulation of epithelial cell death and regeneration. ${ }^{6}$ Previous studies have demonstrated an essential role for IELs against infections caused by certain microorganisms and parasites. CD $8 \alpha \beta^{+} \mathrm{T}$ cells are the major population active against Toxoplasma gondii infection ${ }^{7}$ and markedly contribute to elimination of virus infected intestinal mucosa cells. ${ }^{8}$ Other T cell subsets are also known to contribute to the defence system in the intestinal tract. ${ }^{9}$ On the other hand, IECs can produce various cytokines and play an important role in trafficking and maintenance of IELs. ${ }^{10-12}$ For example, $\alpha_{E} \beta_{7}$ is one of the adhesion molecules that mediate homing of lymphocytes to the gut, and upregulation of its expression has been shown to be completely dependent on epithelial derived TGF- $\beta .{ }^{11}$ Studies with cytokine receptor deficient mice have also pointed to the importance of IEC derived IL-2, IL-7, and IL-15 for IEL development. ${ }^{13} 14$ These observations imply that the IEL-IEC interaction is required to maintain intestinal immunity.

Id proteins are negative regulators of the functions of basic helix-loop-helix (bHLH) transcription factors, which contain a helix-loop-helix domain for protein-protein interaction and a basic region for DNA binding. ${ }^{15}$ Id proteins are involved in many aspects of the immune system, reflecting the central role of bHLH factors (exemplified by E2A gene products E47 and E12) in the differentiation, proliferation, and functions of immune cells. ${ }^{15-21} \mathrm{Id} 2^{-/-}$mice lack lymph nodes, Peyer's patches, ${ }^{17}$ and nasopharyngeal lymphoid tissue ${ }^{18}$ due to a developmental defect of lymphotoxin producing cells that are essential for secondary lymphoid organ development. In addition, Id 2 is required for the development of natural killer

Abbreviations: $b H \mathrm{LH}$, basic helix-loop-helix; IELs, intestinal intraepithelial lymphocytes; LPLs, lamina propria lymphocytes; IECs, intestinal epithelial cells; RT-PCR, reverse transcription-polymerase chain reaction; TCR, T cell receptor; IL, interleukin; TGF- $\beta$, transforming growth factor $\beta$; IFN- $\gamma$, interferon $\gamma$; FCS, fetal calf serum; PBS, phosphate buffered saline; FITC, fluorescein isothiocyanate; PE, phycoerythrin; $\mathrm{mAb}$, monoclonal antibody; 5-FU, 5-fluorouracil; SCF, stem cell factor 
cells, ${ }^{19} \mathrm{CD} 8 \alpha^{+}$dendritic cells, ${ }^{20}$ and Langerhans cells in the skin. ${ }^{20}$ Furthermore, we have shown that Id2 functions as a safeguard against inappropriately increased class switching to IgE that is induced by E2A gene products in response to B cell activation. ${ }^{21}$

Given these findings, we speculated that Id 2 might be involved in the development and/or activation of intestinal lymphocytes. By analysing intestinal lymphocytes of $\mathrm{Id} 2^{-/-}$ mice, we found impairments in their number and in the proportions of their subsets. Alteration of the ability of IECs to produce various cytokines was also noted in $\mathrm{Id} 2^{-1-}$ mice. $\mathrm{Id} 2^{-1-}$ mice exhibited impaired barrier function of the small intestine. Our data provide evidence that Id2 is an important molecule for the organisation of the mucosal defence in the intestine.

\section{METHODS}

Mice

Male Id2 deficient mice, 12-16 weeks of age, of mixed genetic background $(129 / \mathrm{Sv} \times \mathrm{NMRI})$ were used in this study. All mice were maintained under specific pathogen free conditions and all experimental procedures followed the guidelines of School of Medicine, University of Fukui for animal experiments.

\section{Isolation of lymphocytes from intestinal tissues}

IELs, lamina propria lymphocytes (LPLs), and IECs were isolated from individual mice by a standard mechanical dissociation method, as described previously. ${ }^{12}{ }^{22}$ Briefly, after the Peyer's patches as well as fatty tissues and the mesentery were removed from the small intestine, the gut was opened longitudinally and cut into $1 \mathrm{~cm}$ pieces. These pieces were washed in RPMI 1640 (Sigma, St Louis, Missouri, USA) and incubated with stirring in $40 \mathrm{ml}$ of RPMI 1640 containing $1 \mathrm{mM}$ ethylenediamine tetraacetic acid (EDTA; Wako Pure Chemical Industries, Ltd, Japan) at $37^{\circ} \mathrm{C}$ for 30 minutes. The supernatants containing epithelial cells and IELs were collected, and the isolation procedure was repeated two more times. Pooled supernatants were then passed through a $70 \mu \mathrm{m}$ nylon filter to remove cell debris and separated on a discontinuous density gradient of $25 \%, 40 \%$, and $75 \%$ Percoll. IELs at the $40 \% / 75 \%$ interface and IECs at the $25 \% / 40 \%$ interface were collected and extensively washed with RPMI 1640 containing $10 \%$ fetal calf serum (FCS; JRH Biosciences, Kansas, USA).

\section{Flow cytometric analysis and cell sorting}

Freshly isolated IELs and LPLs from individual mice were suspended in phosphate buffered saline (PBS) containing $2 \%$ FCS at $10^{6}$ cells $/ \mathrm{ml}$ and stained in plastic tubes with the following fluorescein isothiocyanate (FITC), phycoerythrin (PE), or biotin conjugated monoclonal antibodies (mAbs) purchased from Pharmingen (San Diego, California, USA): anti-CD3 $\epsilon$ (145-2C11), anti-CD4 (RM4-5), anti-CD8 $\alpha$ $(53-6.7)$, anti-CD8 $\beta \quad(53-5.8)$, anti-CD45R/B220 (RA36B3), anti-49d (R1-2), anti-CD103 (M290), anti-TCR $\beta$ (H57.597), and anti-TCR $\delta$ (GL3). After incubation on ice for 20 minutes, cells were washed twice in PBS containing $2 \%$ FCS and analysed using a FACSCalibur (Becton Dickinson, California, USA) with Cellquest. Forward and side angle scatter were used to exclude dead and aggregated cells. In order to obtain a highly enriched epithelial cell population, Percoll gradient isolated IECs were stained with FITC conjugated anti-I-A ${ }^{\mathrm{b}}$ (AF6-120.1), PE conjugated antiCD3, anti-CD4, anti-CD8 $\alpha$, anti-CD45R/B220 mAb, and

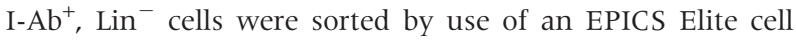
sorter (Coulter Electronics Ltd, Miami, Florida, USA). The purity of the cell population was more than $99 \%$.
RT-PCR and real time PCR analyses of mRNA levels

Total RNA was extracted from IECs using Isogen reagent (Wako) according to the manufacturer's protocol. RNA samples were reverse transcribed (RT) with $5 \mu \mathrm{M}$ random hexamers, $1 \mathrm{mM}$ dNTP, $20 \mathrm{U}$ of RNase inhibitor (Promega Biosciences, San Luis Obispo, California, USA), and $100 \mathrm{U}$ of Moloney murine leukaemia virus reverse transcriptase (Wako) in a volume of $20 \mu \mathrm{l}$ at $42{ }^{\circ} \mathrm{C}$ for 50 minutes. Ids expression was determined by polymerase chain reaction (PCR) using the following primer sets: Id l, 5'-TCA GGA TCA TGA AGG TCG CCA GTG-3' and 5'-TGA AGG GCT GGA GTC CAT CTG GT-3'; Id2, 5' -TCT GAG CTT ATG TCG AAT GAT AGC- $3^{\prime}$ and $5^{\prime}$-CAC AGC ATT CAG TAG GCT CGT GTC GTC3'; Id3, 5'-CCTCTC TAT CTC TAC TCT CCA ACA-3' and 5'-TGA CCA GCG TGT GCT AGC TCT TCA-3'; Id4, 5'-GCG ATA TGA ACG ACT GCT ACA GTC-3' and 5'-ACT TAG CAG TCT GGT CGA CAA CAC-3'. Real time PCR was performed using the double stranded DNA binding dye SYBR Green I with the ABI Prism 7700 system (Applied Biosystems, Foster City, California, USA). The sequences of primers used in this study were as follows: stem cell factor (SCF), 5' -TCT TCA ACT GCT CCT ATT T-3' and 5'-ACT GCT ACT GCT GTC ATT3'; TGF- $\beta 1,5^{\prime}$-CGG GAG GCC AGC CGC GGG AC-3' and 5' GTA ACG CCA GGA ATT GTT GC-3'; IL-6, 5'-GAA CAA CGA TGA TGC ACT TGC AG-3' and 5' -TCC TTA GCC ACT CCT TCT GTG ACT-3'; IL-7, 5' -GCC TGT CAC ATC ATC TGA GTG CC-3' and 5'-CAG GAG GCA TCC AGG AAC TTC TG-3'; IL-15, 5'CTT CTG TCC AGC TAC TCT TCC CCA-3' and 5'-CCA AAC ACA GCA GGA TCC CGT CTT-3'; $\beta$-actin, $5^{\prime}$-CAA CCG TGA AAA GAT GAC CCA GAT C $-3^{\prime}$ and $5^{\prime}$-AGT CCA TCA CAA TGC CTG TGG TAC-3'.

\section{Bone marrow transplantation}

Male C57BL/6 mice (Ly9.2) at eight weeks of age were lethally irradiated $(9.0 \mathrm{~Gy})$ and injected intravenously with

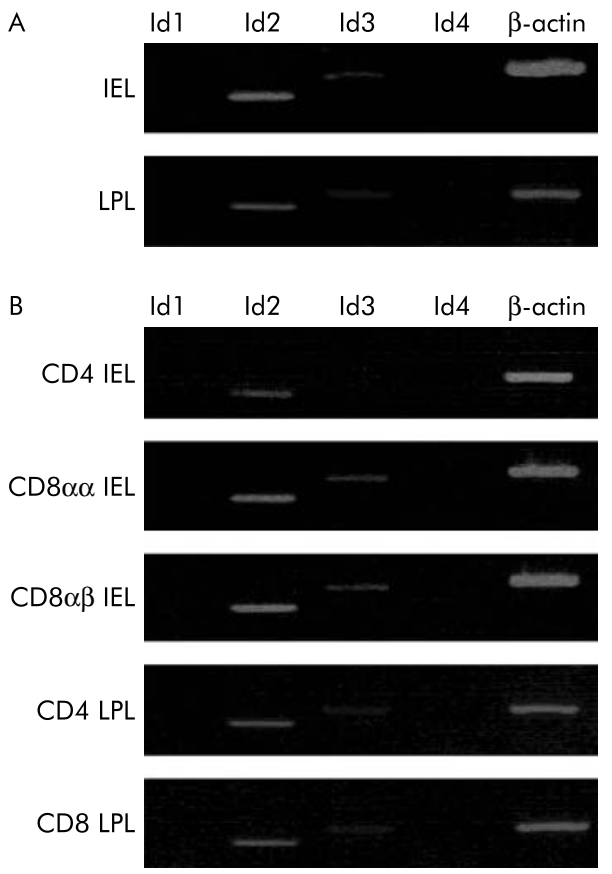

Figure 1 Reverse transcription-polymerase chain reaction (RT-PCR) analysis of gene expression of Ids in intestinal intraepithelial lymphocytes (IELs). (A) Id gene expression in IELs and (B) lamina propria lymphocytes (LPLs) and in their subsets. RNA was prepared from the respective cells as indicated on the left and subjected to RT followed by PCR. Genes analysed are indicated on the top. $\beta$-actin served as an internal loading control. Representative data of three independent experiments are shown. 
$5 \times 10^{6}$ bone marrow cells isolated from $\mathrm{Id} 2^{+/+}$or $\mathrm{Id} 2^{-/-}(129 /$ Sv, Ly9.1) mice. Eight weeks after cell transfer, three colour fluorescence cytometry was used to detect donor derived lymphocytes based on expression of Ly9.1.

\section{5-fluorouracil (5-FU) treatment and counting of endogenous bacterial colonies}

Mice were injected with $800 \mathrm{mg} / \mathrm{kg} 5$-fluorouracil (5-FU) (Sigma) intraperitoneally and mortality was monitored everyday. The livers and spleens were removed and homogenised with $5 \mathrm{ml}$ PBS. Homogenates were serially diluted and spread on agar plates containing the MacConkey medium to detect enterobacteria (Kyokuto, Tokyo, Japan).
Colony numbers were counted after incubation for 24 hours at $37^{\circ} \mathrm{C}$.

\section{Statistical analysis}

The Student's $t$ test was used to determine the significance of differences. A p value of less than 0.05 was taken as significant.

\section{RESULTS}

Small intestinal IELs express Id 2 mRNA

As mice deficient in Id genes show various defects in the immune system, we considered whether Id 2 plays some role in small intestinal lymphocytes which constitute a large part
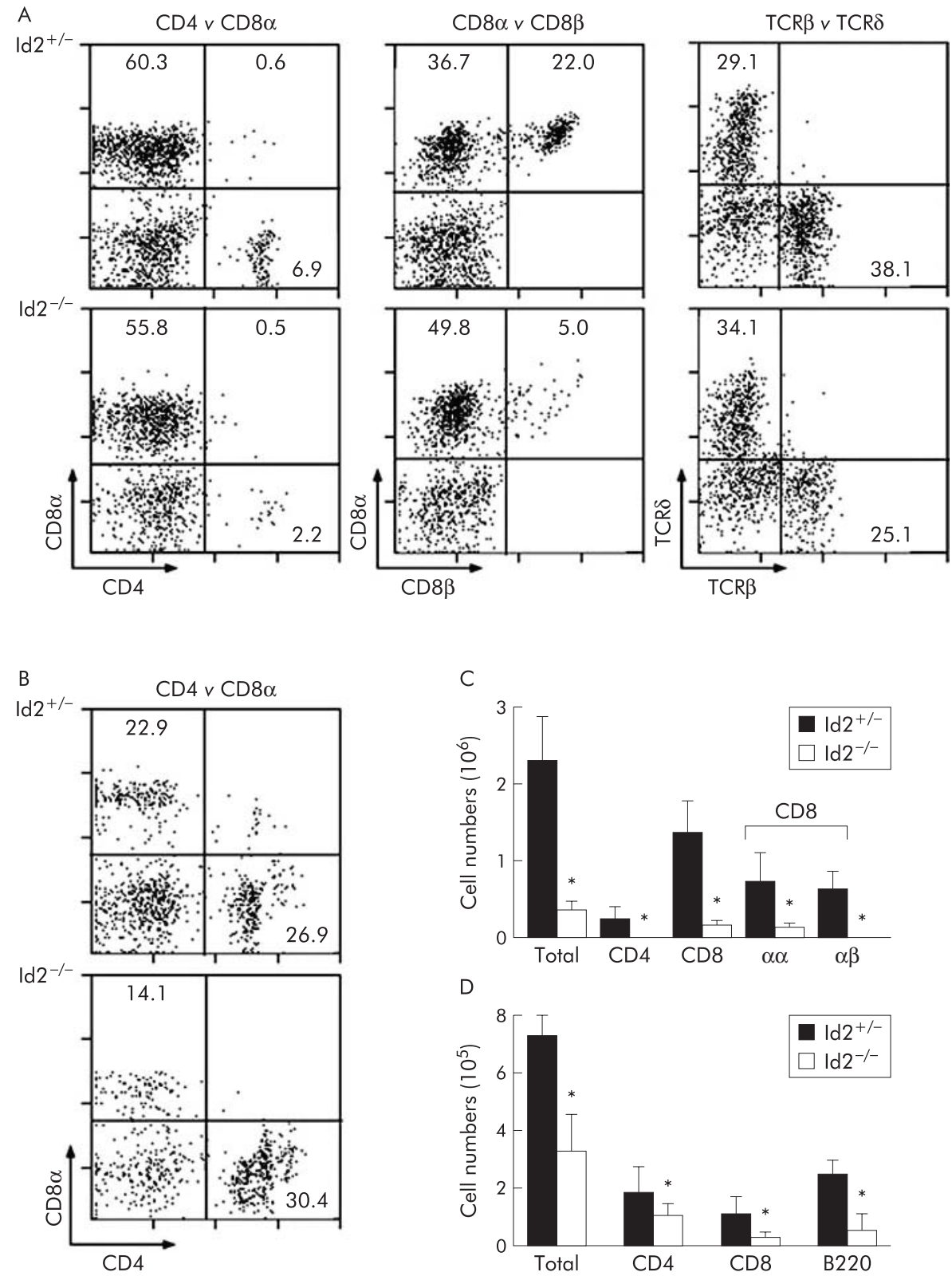

Figure 2 Decreased numbers and aberrant proportions of intestinal lymphocytes in $\mathrm{ld} 2^{-1-}$ mice. (A) Flow cytometric analysis of intestinal T cell subsets of intestinal intraepithelial lymphocytes (IELs). Representative data from 4-6 independent experiments are shown. Intestinal lymphocytes were isolated and analysed for the proportion of each T cell subset. The cell surface markers used are indicated at the bottom left corners. Percentages of subsets are shown in the respective fractions. Genotypes are indicated on the left. (B) Flow cytometric analysis of intestinal T cell subsets of lamina propria lymphocytes (LPLs). Details as described in (A). (C) Cell numbers of the respective IEL subsets. The absolute number of each subset was obtained by multiplying the percentage of cells by the total number of cells in the respective populations. Results are represented as mean (SEM) number. Each number represents the mean of $4-6$ animals per group. ${ }^{*} p<0.05$, as determined by the Student's two tailed $t$ test. (D) Cell numbers of the respective LPL subsets. Details as described in (C). 
of the immune system in mammals. To address this question, we first used RT-PCR to examine expression of Id genes in IELs and found selective expression of Id 2 in these cells (fig 1A). We further sorted various $\mathrm{T}$ cell subsets from IELs and LPLs of wild-type mice and examined mRNA expression of Id genes in these subsets. As shown in fig 1B, all subsets of IELs $\left(\mathrm{CD} 8 \alpha \alpha^{+}, \mathrm{CD} 8 \alpha \beta^{+}\right.$, and $\mathrm{CD} 4^{+} \mathrm{T}$ cells) mainly expressed Id 2 mRNA, and also weakly expressed Id3. In addition, although $\mathrm{T}$ cell subsets of LPLs $\left(\mathrm{CD}^{+}\right.$and $\mathrm{CD}^{+} \mathrm{T}$ cells) expressed Id 2 and Id3, expression was barely detectable. These results strongly suggest that Id 2 is profoundly involved in the development, survival, and/or activation of IELs.

\section{Id2 $2^{-/-}$mice have decreased numbers of $\mathrm{CD}^{+}$and $\mathrm{CD4}^{+} \mathrm{T}$ lymphocytes in IELs}

We next determined the total number and proportions of IEL subsets in $\mathrm{Id} 2^{-/-}$mice and found dramatic alterations. The absolute number of small intestinal IELs in $\mathrm{Id} 2^{-/-}$mice was $0.36(0.10) \times 10^{6}$ cells while that in $\mathrm{Id}^{+/-}$mice was 2.29 $(0.57) \times 10^{6}$ cells, indicating a sevenfold reduction in Id null mice. Population analysis using cell surface markers showed that the percentages of cells positive for CD4, CD8 $\beta$, and TCR $\beta$ were decreased by 3.2-, 5.6-, and 2.0 -fold in Id $2^{-1-}$ mice, respectively, indicating that the proportions of thymus dependent $\mathrm{CD}^{+}$and $\mathrm{CD} 8 \alpha \beta^{+}$subsets were reduced (fig $2 \mathrm{~A}$ ). The absolute numbers of various subsets of IELs of Id $2^{-1-}$ mice were $0.02(0.02), 0.16(0.06), 0.15(0.05)$, and 0.02 $(0.01) \times 10^{6}$ cells/intestine for $\mathrm{CD}^{+}, \mathrm{CD} 8^{+}, \mathrm{CD} 8 \alpha \alpha^{+}$, and $\mathrm{CD} 8 \alpha \beta^{+} \mathrm{T}$ cell subsets, respectively (fig $2 \mathrm{C}$ ). These data reveal that the subset cell numbers were decreased by 12.0-, 8.5-, $4.9-$, and 38.0-fold compared with those of control $\mathrm{Id}^{+/-}$ littermates $(0.24(0.16), 1.36(0.42), 0.74(0.37)$, and 0.66 $(0.21) \times 10^{6}$ cells/intestine for $\mathrm{CD}^{+}, \mathrm{CD} 8^{+}, \mathrm{CD} 8 \alpha \alpha^{+}$, and $\mathrm{CD} 8 \alpha \beta^{+}$subsets, respectively). A similar tendency was also observed for LPLs, along with a decrease in the number of $\mathrm{B} 220^{+}$cells, although the $\mathrm{CD} 4^{+} \mathrm{T}$ cell subset of LPLs showed only a slight tendency to decrease (fig $2 \mathrm{~B}, 2 \mathrm{D}$, and data not shown). These results indicate that Id 2 deficiency affects IELs more severely than LPLs, and on thymus dependent subsets than on thymus independent subsets.

\section{Impairment of IELs of Id2 ${ }^{-/-}$mice is intrinsic to bone marrow derived cells}

To obtain insight into the cause of impairment of IELs in $\mathrm{Id} 2^{-/-}$mice, we next performed bone marrow transplantation experiments and analysed the $\mathrm{T}$ cell subsets in the intestinal mucosa. Transplantation was conducted from 129/ Sv mice (Ly9.1) to C57/BL/6 mice (Ly9.2), and donor derived cells were discriminated from recipient cells by utilising the Ly9 isotype system. ${ }^{17}$ In mice that received bone marrow cells from $\mathrm{Id} 2^{-1-}$ mice, there was a fourfold reduction in the total number of IELs compared with that in mice that received $\mathrm{Id} 2^{+/+}$bone marrow cells (fig 3 ). In addition, the proportions of the various $\mathrm{T}$ cell subsets in IELs recapitulated the phenotype found in $\mathrm{Id} 2^{-1-}$ mice (fig $3 \mathrm{~A}$ ). Taken together, these results demonstrate that the defects found in IELs of $\mathrm{Id} 2^{-/-}$mice are intrinsic to bone marrow derived cells.

\section{Expression of $\alpha_{E}$ integrin in CD $8 \alpha \beta^{+}$IELs is impaired in} Id $2^{-l-}$ mice

We next evaluated expression of various adhesion molecules, including $\beta_{2}$ integrin (CD18), $\alpha_{4}$ integrin (CD49d), ICAM-1 (CD54), and $\alpha_{\mathrm{E}}$ integrin (CD103) as they are known to be involved in the development and trafficking of $\mathrm{CD}^{+} \mathrm{T}$ cells in the intestinal epithelium..$^{23}{ }^{24}$ As shown in fig $4 \mathrm{~A}$, the $\mathrm{CD} 4^{+} \mathrm{T}$ cell subset of IELs isolated from $\mathrm{Id}^{-/-}$mice showed a significantly reduced level of $\alpha_{\mathrm{E}}$ integrin expression. In addition, the $\operatorname{CD} 8 \alpha \beta^{+} \mathrm{T}$ cell subset of IELs also showed a reduction of $\alpha_{\mathrm{E}}$ integrin expression in a biphasic manner while CD $8 \alpha \alpha^{+}$IELs of $\mathrm{Id} 2^{-/-}$mice showed little alteration in expression. On the other hand, we could not find any significant differences in expression of $\alpha_{4}$ integrin (CD49d), $\beta_{2}$ integrin (CD18), or ICAM-1 (CD54) in T cell subsets between wild-type and $\mathrm{Id} 2^{-/-}$mice (fig $4 \mathrm{~B}$, and data not shown). These results suggest that $\alpha_{\mathrm{E}}$ integrin expression is selectively impaired in thymus dependent IELs of $\mathrm{Id} 2^{-/-}$ mice which is consistent with the observation that impairment of IELs of $\mathrm{Id} 2^{-/-}$mice was more severe in thymus dependent IELs than in thymus independent IELs.

\section{mRNA expression of IEC derived cytokines is decreased in Id $2^{-/-}$mice}

IECs have been shown to produce several cytokines ${ }^{10}{ }^{12}$ and it was important to examine whether the decrease in IELs affected the cytokine production ability of IELs in $\mathrm{Id} 2^{-/-}$ mice. To compare cytokine mRNA expression in wild-type and $\mathrm{Id} 2^{-/-}$mice, we isolated IECs and carried out cytokine specific real time PCR. As shown in fig 5, expression of IL-7,
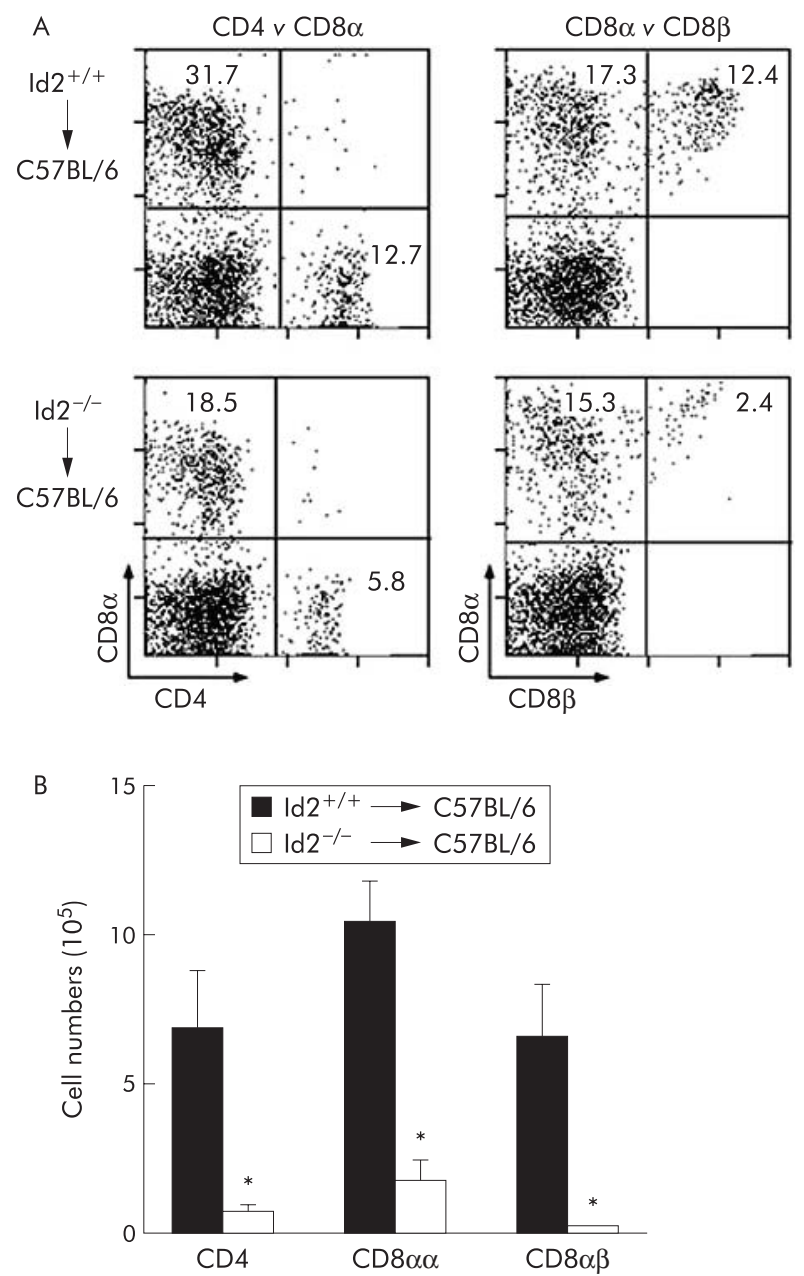

Figure 3 Phenotypic analysis of intestinal intraepithelial lymphocytes (IELs) after bone marrow transplantation. (A) Flow cytometric analysis of IELs of C57BL/6 mice (Ly 9.2) mice that were reconstituted with bone marrow cells of $\mathrm{Id} 2^{+/+}$or $\mathrm{Id} 2^{-/-}$mice (Ly 9.1). IELs of donor origin were analysed for cell surface marker expression, as indicated by gating Ly $9.1^{+}$cells. Percentages of cells with a given phenotype are shown. Representative data of four mice per group are shown. (B) Absolute number of each subset was obtained by multiplying the percentage of each subpopulation by the total number of viable cells. Results are expressed as mean (SEM) number. Each number represents the mean of four animals per group. ${ }^{*} p<0.05$, as determined by the Student's two tailed $t$ test. 
A

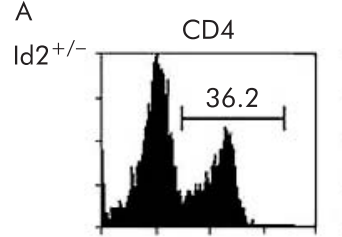

$\mathrm{Id} 2^{-}$

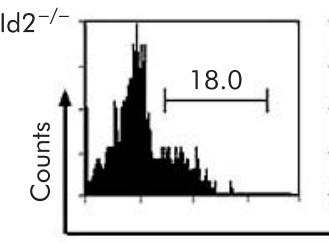

$\operatorname{CD} 8 \alpha \alpha$
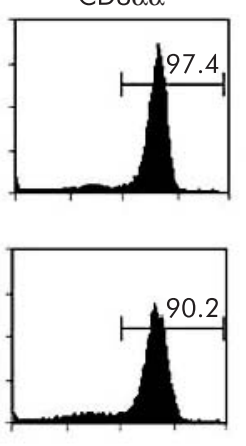

$\alpha_{E}$ integrin (CD103)
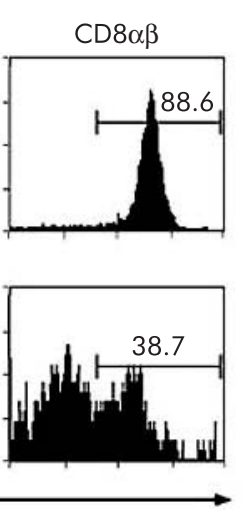

B

$1 \mathrm{~d} 2^{+/}$

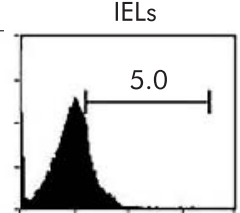

$\operatorname{ld} 2^{-/}$

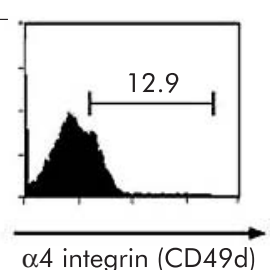

Figure 4 Reduced expression of $\alpha_{E}$ integrin on thymus derived intestinal intraepithelial lymphocytes (IELs). (A) IELs were isolated and stained with phycoerythrin conjugated antimouse CD4, antimouse $\mathrm{CD} 8 \alpha$, fluorescein isothiocyanate (FITC) conjugated antimouse CD8 $\beta$, or biotin conjugated antimouse CD103 monoclonal antibody (mAb). After gating of cells positive for the respective mAbs, the percentages of $\alpha_{E}$ integrin positive cells in the histogram were calculated. (B) Isolated IELs were stained with FITC conjugated antimouse CD49d mAb. Cell populations are indicated on the top of each panel. Representative data of 4-6 independent experiments are shown.

IL-15, and TGF- $\beta 1$ mRNAs in isolated IECs was decreased in $\mathrm{Id} 2^{-/-}$mice compared with those in IECs of control littermates. There was no significant difference in expression of SCF between the two groups of mice.

High susceptibility of 5 -FU treatment in Id $2^{-/-}$mice The impaired number of IELs and cytokine production by IECs of $\mathrm{Id} 2^{-1-}$ mice prompted us to examine intestinal barrier function of $\mathrm{Id} 2^{-1-}$ mice. To this end, we utilised 5-FU treatment of mice. It is well known that the cytotoxicity of 5-FU disrupts the epithelial barrier and allows intestinal bacteria to invade, which can lead to translocation of intestinal bacteria to the liver and lethal infection. ${ }^{25-28}$ This system allowed us to evaluate intestinal barrier function against invading bacteria. After 5-FU treatment, all of wildtype control mice survived during the experimental period. In contrast, only $37.5 \%$ of $\mathrm{Id} 2^{-/-}$mice survived to day 12 after treatment (fig 6A). In accordance with these observations, the numbers of enterobacteria in the liver and spleen of $\mathrm{Id} 2^{-/-}$mice were substantially increased compared with wild-type control mice after administration of 5-FU (fig 6B). These results demonstrate that $\mathrm{Id} 2^{-/-}$mice are susceptible to 5-FU treatment and have impaired intestinal barrier function.

\section{DISCUSSION}

In this study, we have shown that Id2 is required for IELs to constitute the normal mucosal barrier system in the intestine and thus demonstrated a novel aspect of the in vivo function of Id2, which has been shown to be required for differentiation of various cell types in the immune system. Consistent with our finding of $\operatorname{Id} 2$ expression in IELs, we found a generalised reduction in the number of IELs in $\mathrm{Id} 2^{-/-}$mice. The reduction was more pronounced in thymus derived $\mathrm{CD} 4^{+}$ and CD $8 \alpha \beta^{+}$T cell subsets, leading to alteration of the subset profile. These two subsets of IELs in $\mathrm{Id} 2^{-/-}$mice were also found to express decreased levels of $\alpha_{\mathrm{E}}$ integrin. Furthermore, bone marrow transplantation experiments demonstrated that the defects were intrinsic to bone marrow derived cells of $\mathrm{Id} 2^{-/-}$mice. In addition to the phenotypes found for IELs, IECs of Id $2^{--/-}$mice were found to have an impaired ability to produce cytokines that are thought to play an important role in maintaining the mucosal defence in the intestinal tract. Thus our findings strengthen the idea that $\mathrm{Id} 2$ is an essential molecule for establishment of the immune system that protects our bodies from invading microorganisms.
The decrease in the number of lymphocytes observed in Id $2^{-1-}$ mice is a phenomenon that selectively affects IELs because there is no such impairment in splenocytes or thymocytes of $\mathrm{Id} 2^{-/-}$mice. ${ }^{17}$ It is currently not known why IELs of Id $2^{-1-}$ mice are decreased as a whole, irrespective of the $\mathrm{T}$ cell subset, although it is clear that the phenotypes of IELs in Id $2^{-1-}$ mice are derived from bone marrow cells. The fact that Id 2 is the predominantly expressed member of the Id gene family, expressed in each T cell subset of IELs, implies a distinct role for Id 2 in the development, survival, and/or proliferation of lymphocytes in the intestine. As Id proteins negatively regulate the function of E proteins, type A bHLH transcription factor, ${ }^{29}$ the balance between the activities of Id 2 and E proteins may be important for the development and/or maintenance of IELs.

We observed selectively diminished expression of $\alpha_{\mathrm{E}}$ integrin in $\mathrm{CD}^{+}$and $\mathrm{CD} 8 \alpha \beta^{+}$IELs of $\mathrm{Id} 2^{-/-}$compared with other adhesion molecules analysed. Adhesion molecule play an indispensable role in maintaining the intestinal immune cells, and $\alpha_{\mathrm{E}} \beta_{7}$ integrin mediates the binding of IELs to IECs through interaction with E-cadherin, a membrane protein previously known for its role in the homophilic interactions

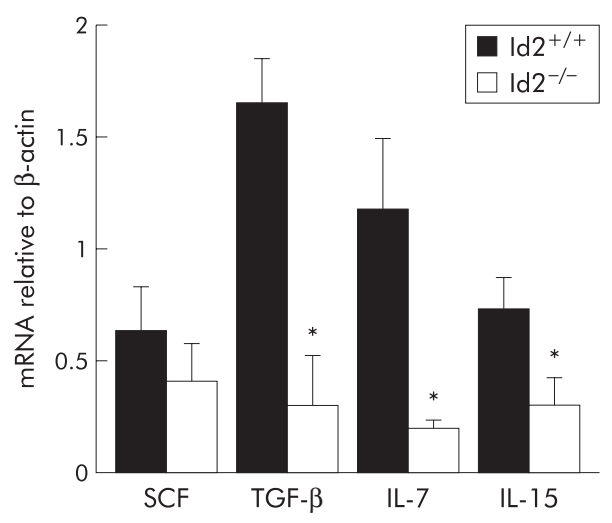

Figure 5 Real time polymerase chain reaction (PCR) analysis of cytokine expression in intestinal epithelial cells (IECs). RNA was prepared from the IECs of Id $2^{+/+}$or $\mathrm{Id} 2^{-/-}$mice and subjected to reverse transcription followed by real time PCR, as described in the methods section. Results are expressed as mean (SEM) number. Each bar represents the mean of three animals per group. ${ }^{*} \mathrm{p}<0.05$, as determined by the Student's two tailed $t$ test. SCF, stem cell factor; IL, interleukin; TGF- $\beta$, transforming growth factor $\beta$. 

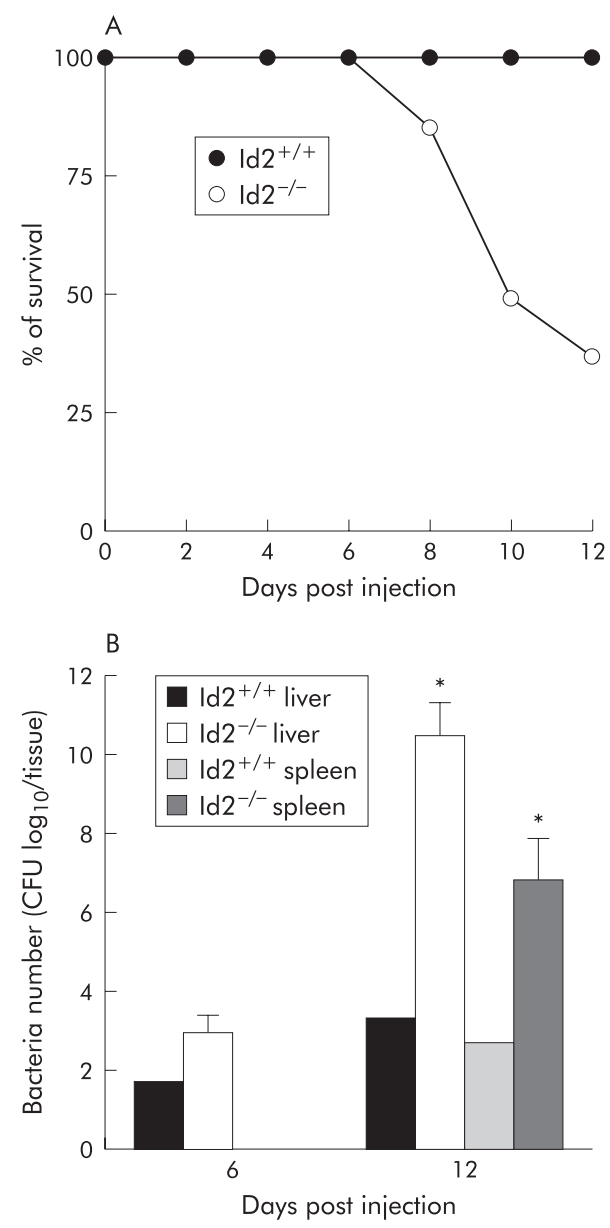

Figure 6 Increased susceptibility of Id2 deficient mice after 5 -fluorouracil $(5$-FU) treatment. (A) Survival rate of mice after 5-FU treatment. Id $2^{+/+}$and $I d 2^{-/-}$mice were treated intraperitoneally with $800 \mathrm{mg} / \mathrm{kg} 5$-FU. Eight mice were used in each group. (B) Translocation of endogenous bacteria from the intestine to the liver and spleen in mice treated with 5-FU. Numbers of bacteria in the liver and spleen of $1 \mathrm{~d} 2^{+/+}$ and $\mathrm{Id} 2^{-1-}$ mice were determined on the indicated days after intraperitoneally injection of $800 \mathrm{mg} / \mathrm{kg} \mathrm{5-FU.} \mathrm{Values} \mathrm{represent} \mathrm{mean}$ (SEM) of three animals. ${ }^{*} p<0.05$, as determined by the Student's two tailed $t$ test.

of adherent junctions. ${ }^{30}$ Moreover, $\alpha_{\mathrm{E}}$ integrin deficient mice show a substantially reduced number of IELs. ${ }^{23}{ }^{24}$ As E-cadherin expression in IECs of $\mathrm{Id}^{-/-}$mice is normal (unpublished observations), impaired expression of $\alpha_{\mathrm{E}}$ integrin in thymus derived IEL subsets can explain, at least in part, why the number of thymus derived IELs is more severely reduced than that of extrathymically derived subsets, and supports the notion that $\alpha_{E} \beta_{7}$ integrin is important for IELs. Id 2 may be involved in regulating $\alpha_{\mathrm{E}}$ integrin expression by inhibiting the activity of some bHLH factor that suppresses expression in these cell types, although the detailed mechanism remains unclear.

IEC derived cytokines, including IL-6, IL-7, IL-15, and TGF- $\beta$, are indispensable for the development and survival of lymphocytes in the intestinal tract, showing distinct but partly overlapping effects on subpopulations of intestinal lymphocytes. ${ }^{10} 3132$ Thus decreased gene expression of these cytokines in IECs of Id $2^{-/-}$mice may reflect the generalised impairment of IECs of Id $2^{-1-}$ mice. However, the defective features of IELs of Id $2^{-1-}$ mice were essentially reconstituted in wild-type mice that received bone marrow transplants from $\mathrm{Id} 2^{-1-}$ mice. These findings suggest that the defect is intrinsic to the immune cells of $\mathrm{Id} 2^{-1-}$ mice. It is therefore plausible that IECs of $\mathrm{Id} 2^{-/-}$mice per se have a defect in producing these cytokines, although we cannot completely rule out the possibility that impaired IELs affect the functions of IECs of Id $2^{-1-}$ mice. In relation to these observations, we analysed the Th1/Th2 balance in IELs of $\mathrm{Id} 2^{-/-}$mice because splenic $\mathrm{CD} 4^{+} \mathrm{T}$ cells of $\mathrm{Id} 2^{-/-}$mice are skewed to Th2 cells. ${ }^{33}$ Although we observed a higher proportion of IL-4 producing $\mathrm{CD} 4^{+} \mathrm{T}$ cells, we did not see a difference in IFN- $\gamma$ producing $\mathrm{CD}^{+}{ }^{+} \mathrm{T}$ cells in IELs (data not shown). These findings may suggest that differentiation and/or maintenance of $\mathrm{CD}^{+} \mathrm{T}$ cells in the intestine is different from that in the spleen. Currently, it is unclear whether the increased proportion of Th2 cells in the intestine contributes to generation of impaired IECs of $\mathrm{Id} 2^{-1--}$ mice. Further investigations are required to characterise IECs of $\mathrm{Id} 2^{-/-}$mice.

The gastrointestinal tract serves as a major site for primary infection as well as for the entry of pathogens, and the immune response at the mucosal surface provides the first barrier to invading pathogens. As IELs have a predominant role in excluding pathogens in the intestine through their cytolytic activity and/or inflammatory cytokine production, ${ }^{6-10}$ we assessed the physiological meaning of impaired number of IELs and cytokine production of IECs in Id 2 null mutant mice. Utilising 5-FU treatment, a decreased survival rate and enhanced numbers of enterobacteria in the liver and spleen of $\mathrm{Id} 2^{-/-}$mice (fig 6) strongly suggested that intestinal barrier function is severely impaired in mutant mice. In this regard, Id2 null mutant mice may provide a good model system for studying gastrointestinal infection. In addition, $\mathrm{Id} 2^{-/-}$mice are unique among other gene deficient mice that show a defect in intestinal lymphocytes, such as $\mathrm{IL}^{-7^{-/}}$, IL-7R $\alpha^{-1-}$, and IL-2R $\beta^{-1-}$ mice, ${ }^{34-37}$ in that $\mathrm{Id} 2$ null mice show impairment of lymphocytes rather selectively in the intestine, a generalised reduction of IELs, and a stronger effect on thymus derived IEL subsets. Further investigations using $\mathrm{Id} 2^{-/-}$mice may disclose in detail the developmental pathway of lymphocytes in the intestine.

\section{ACKNOWLEDGEMENTS}

This work was supported by Grants-in Aid from the Ministry of Education, Culture, Sports, Science, and Technology, Japan, (YY \#13470034, \#13037016, and \#15390102). J-KK was supported by grants from the Japan Society for the Promotion of Science.

\section{Authors' affiliations}

J-K Kim, M Takeuchi, Y Yokota, Department of Molecular Genetics, School of Medicine, University of Fukui, 23-3 Shimoaizuki, Matsuoka, Fukui 910-1193, Japan

\section{REFERENCES}

1 Kaufmann SH. $\gamma / \delta$ and other unconventional lymphocytes: what do they see and what do they do? Proc Natl Acad Sci U S A 1996;93:2272-9.

2 Rocha B, Vassalli P, Guy-Grand D. Thymic and extrathymic origins of gut intraepithelial population in mice. J Exp Med 1994;180:681-6.

3 Poussier $\mathbf{P}$, Julius $M$. Thymus independent T cell development and selection in the intestinal epithelium. Annu Rev Immunol 1994; 12:521-53.

4 Beagley KW, Husband AJ. Intraepithelial lymphocytes: origins, distribution, and function. Crit Rev Immunol 1998;18:237-54.

5 Das G, Janeway CA. MHC specificity of ilELs. Trends Immunol 2003;24:88-93.

6 Fujihashi K, Yamamoto M, McGhee JR, et al. Function of $\alpha \beta \mathrm{TCR}^{+}$intestinal intraepithelial lymphocytes: Th- 1 and Th-2 type cytokine production by $\mathrm{CD} 4^{+} \mathrm{CD} 8^{-}$and $\mathrm{CD} 4^{+} \mathrm{CD} 8^{+} \mathrm{T}$ cells for helper activity. Int Immunol 1993:5:1473-81.

7 Buzoni-Gatel D, Debbabi H, Moretto $M$, et al. Intraepithelial lymphocytes traffic to the intestine and enhance resistance to Toxoplasma gondii oral infection. J Immunol 1999; 162:5846-52.

8 Muller S, Buhler-Jungo M, Mueller C. Intestinal intraepithelial lymphocytes exert potent protective cytotoxic activity during an acute virus infection. $\mathrm{J}$ Immunol 2000; 164:1986-94.

9 McDonald V, Robinson HA, Kelly JP, et al. Immunity to Cryptosporidium muris infection in mice expressed through gut $\mathrm{CD} 4^{+}$intraepithelial lymphocytes. Infect Immun 1996;64:2556-62. 
10 Yoshikai Y. The interaction of intestinal epithelial cells and intraepithelial lymphocytes in host defense. Immunol Res 1999;20:219-35.

11 Shibahara T, Si-Tahar M, Shaw SK, et al. Adhesion molecules expressed on homing lymphocytes in model intestinal epithelia. Gastroenterology 2000; 118:289-98.

12 Yamamoto M, Fujihashi K, Kawabata K, et al. A mucosal intranet: intestinal epithelial cells down-regulated intraepithelial, but not peripheral T lymphocytes. J Immunol 1998;160:2188-96.

13 Porter BO, Malek TR. IL-2R $\beta /$ IL-7R $\alpha$ doubly deficient mice recapitulate the thymic and intraepithelial lymphocyte (IEL) developmental defects of gamma $\mathrm{c}^{-1-}$ mice: roles for both IL-2 and IL-15 in CD8 $\alpha \alpha$ IEL development. J Immunol 1999; 163:5906-12.

14 Inagaki-Ohara K, Nishimura H, Mitani A, et al. Interleukin-15 preferentially promotes the growth of intestinal intraepithelial lymphocytes bearing $\gamma \delta$ T cell receptor in mice. Eur J Immunol 1997;27:2885-91.

15 Yokota Y. Id and development. Oncogene 2001;20:8290-8.

16 Rivera R, Murre $C$. The regulation and function of the Id proteins in lymphocyte development. Oncogene 2001;20:8308-16.

17 Yokota Y, Mansouri A, Mori S, et al. Development of peripheral lymphoid organs and natural killer cells depends on the helix-loop-helix inhibitor Id2. Nature 1999;397:702-6.

18 Fukuyama S, Hiroi T, Yokota Y, et al. Initiation of NALT organogenesis is independent of the IL-7R, LT $\beta R$, and NIL signaling pathways but requires the Id 2 gene and $\mathrm{CD}^{-}{ }^{-} \mathrm{CD} 4^{+} \mathrm{CD} 45^{+}$cells. Immunity 2002;17:1-10.

19 Ikawa T, Fujimoto S, Kawamoto $\mathrm{H}$, et al. Commitment to natural killer cells requires the helix-loop-helix inhibitor Id2. Proc Natl Acad Sci U S A 2001;98:5164-9.

20 Hacker C, Kirsch RD, Ju XS, et al. Transcriptional profiling identifies Id2 function in dendritic cell development. Nat Immunol 2003;4:380-6.

21 Sugai M, Gondo H, Kusunoki T, et al. Essential role of Id2 in negative regulation of IgE class switching. Nat Immunol 2003;4:25-9.

22 Kim JK, Takahashi I, Kai Y, et al. Influence of the enterotoxin on mucosa intranet: selective inhibition of extrathymic T cell development in intestinal intraepithelial lymphocyte by oral exposure to heat-labile toxin. Eur J Immunol 2001;31:2960-9.

23 Schon MP, Arya A, Murphy EA, et al. Mucosal T lymphocyte numbers are selectively reduced in integrin $\alpha_{\mathrm{E}}(\mathrm{CD} 103)$-deficient mice. J Immunol $1999 ; 162: 6641-9$
24 Huleatt JW, Lefrancois L. $\beta 2$ integrins and ICAM-1 are involved in establishment of the intestinal mucosal T cell compartment. Immunity 1996;5:263-73

25 liji K, Potten CS. Further studies on the response of intestinal crypt cells of different hierarchical status to eighteen different cytotoxic agents. $\mathrm{Br} J$ Cancer 1987;55:113-23.

26 Nomoto K, Yokomura T, Nomoto K. Prevention of 5-fluorouracil-induced infection with indigenous Escherichia coli in tumor-bearing mice by nonspecific immunostimulation. Can J Microbiol 1992;38:774-8.

27 Tancrede $\mathrm{CH}$, Andremont $\mathrm{AO}$. Bacterial translocation and gram-negative bacteria in patients with hematological malignancies. $J$ Infect Dis 1985; 152:99-103.

28 Itoh N, Nishimura $\mathrm{H}$, Matsuguchi T, et al. CD $8 \alpha$-deficient mice are highly susceptible to 5 -fluorouracil-induced lethality. Clin Diagn Lab Immunol 2002;9:550-7.

29 Massari ME, Rivera RR, Voland JR, et al. Characterization of ABF-1, a novel basic helix-loop-helix transcription factor expressed in activated $B$ lymphocytes. Mol Cell Biol 1998; 18:3130-9.

30 Cepak KL, Shaw SK, Parker CM, et al. Adhesion between epithelial cells and T lymphocytes mediated $\mathrm{E}$-cadherin and an integrin $\alpha_{\mathrm{E}} \beta_{7}$. Nature 1994;372:190-3

31 Brunner T, Arnold D, Wasem C, et al. Regulation of cell death and survival in intestinal intraepithelial lymphocytes. Cell Death Differ 2001;8:706-14.

32 Hirose K, Suzuki H, Nishimura H, et al. Interleukin-15 may be responsible for early activation of intestinal intraepithelial lymphocytes after oral infection with Listeria monocytogenes in rats. Infect Immun 1998;66:5677-83.

33 Kusunoki T, Sugai $M$, Katakai $T$, et al. $T_{H} 2$ dominance and defective development of a CD8 ${ }^{+}$dendeitic cell subset in Id2-deficient mice. J Allergy Clin Immunol 2003;111:136-42.

34 Suzuki H, Duncan GS, Takimoto H, et al. Abnormal development of intestinal intraepithelial lymphocytes and peripheral natural killer cells in mice lacking in the IL-2 receptor $\beta$ chain. J Exp Med 1997; 185:499-505.

35 He YW, Malek TR. Interleukin-7 receptor $\alpha$ is essential for the development ${ }^{+} \mathrm{T}$ cells, but not natural killer cells. J Exp Med 1996; 184:289-93.

36 Maki K, Sunaga S, Komagata Y, et al. Interleukin-7 receptor-deficient mice lack $\gamma \delta$ T cells. Proc Natl Acad Sci U S A 1996;93:7172-7.

37 Puddington L, Olson S, Lefrancosis L. Interactions between stem cell factor and c-Kit are required for intestinal immune system homeostasis. Immunity 1994; 1:733-9.

\section{GI SNAPSHOT}

Answer

From question on page 477

The surgical decision was to adopt a conservative approach and not to proceed to a laparotomy. Subsequent plain abdominal radiographs demonstrated the passage of the blades into the sigmoid colon and thereafter they passed rectally, uneventfully!

The majority (80-90\%) of foreign bodies entering the gastrointestinal tract pass uneventfully, especially if they pass through the gastric pylorus and traverse the duodenal sweep. The remaining $20 \%$ require either endoscopic or surgical removal.

In the case described, the passage of blades beyond the pylorus into the small bowel resulted in no ill effect and the uneventful passing of all three blades per rectum. We have shown that once sharp objects have passed beyond the pylorus, an expectant approach may be safely adopted provided the patient is under close medical observation. The human body has developed many strategies to deal with a range of environmental insults, none perhaps more challenging than the safe passage of shaving blades through the gastrointestinal tract! 